of AT-TPO varied within the norm with a prevalence of in the normal thyroid parenchyma children group and indicated the potential threat of thyroid pathology developing in this group and violation of the processes of iodine oxidation. The values of TCG, T3 in both groups tended to be lower bound when the level T4 corresponded to the upper value of the reference values. The minimum thyroid deficiency was observed in the children thyroid gland hyperplasia, in particular, the TSH level ranged from 2.5 to $4.0 \mathrm{MU} / \mathrm{l}$, the TTG/fT4 index ranged from 0.19 to 0.29 .

Conclusion Recommended the appointment of correction with iodine-selenium supplementation in the children with thyroid gland morphometric changes, which identified risk factors on endemic goiter and minimal thyroid failure

\section{P307 DISSEMINATION OF GOOD PRACTICE: AN ITALIAN PROJECT TO CONTRAST CYBERBULLYING}

${ }^{1}$ Giuliana Pulvirenti*, ${ }^{2}$ Marco Flavio Michele Vismara, ${ }^{3}$ Stefano Alessandro Vismara, ${ }^{4}$ Roberta Elisabetta Vismara, ${ }^{5}$ Viviana Meschesi, ${ }^{6}$ Valeria Moroni, ${ }^{7}$ Serena Maria Lavano, ${ }^{8}$ Joseph Toaff, ${ }^{9}$ Umberto Rapetto, ${ }^{6}$ Riccardo Cemicetti, ${ }^{10}$ Chiara Settanni, ${ }^{11}$ Barbara Zepponi, ${ }^{12}$ Nicola Landolfi, ${ }^{13}$ Antonio Valentini, ${ }^{14}$ Rosa Marotta. ${ }^{1}$ CRISCAT (International Research Center for Theoretical and Applied Cognitive Sciences) University of Messina and Universitary Consortium of Eastern Mediterranean, Noto, Italy; ${ }^{2}$ Pathological Anatomy Unit, Magna Græcia University, Catanzaro, Italy; ${ }^{3}$ Ente Ospedaliero Cantonale del Ticino, Ospedale Regionale di Locarno 'La Carità', Unità di Pediatria, Locarno, Switzerland; ${ }^{4}$ Università di Torino, facoltà di Psicologia, Turin, Italy; ${ }^{5}$ M.I.U.R.(Italian Ministry of Education, University and Research), Rome, Italy; ${ }^{6}$ Informatica and Biomedicina srls, Rome, Italy; ' Dipartimento di Scienze della Salute, Life Science and Technology Graduate School, Magna Graecia University, Catanzaro, Italy; ${ }^{8}$ Communication Science and Media Consulting, Versailles, France; ${ }^{9} \mathrm{HKAO}$ Consulting, Rome, Italy; ${ }^{10}$ Settanni Consulting Group, Rome, Italy; " IIMAGO Psychotherapy Clinic, Rome, Italy; ${ }^{12}$ Life Sciences and Healthcare Lawyer, Rome, Italy; ${ }^{13}$ 'Caravaggio' medical center, Toxicology Unit, Rome, Italy; ${ }^{14}$ Dipartimento di Scienze Mediche e Chirurgiche, Magna Graecia University, Catanzaro, Italy

\subsection{6/archdischild-2019-epa.656}

Introduction The way children use the Internet and mobile technologies has changed in the last few years according to the Digital Agenda for Europe. The growing phenomenon of cyberbullying is among the risks associated with the increasing Internet use. Methods of access and use by teenagers are highly important factors in assessing those risks.

Materials and methods In order to fight cyberbullying we have developed a strategy with the approval of the Department of Medical and Surgical Sciences of the Magna Graecia University of Catanzaro consisting in three phases: a) Data collection and review b) Critical analysis c) Dissemination.

a) We expanded our previous study (2017) with a new cluster; a questionnaire specifically developed to investigate cyberbullying and cyber-grooming among the teenage population. This new cluster, proposed by the 'Cyber Expert' program created by the Spanish Policia Nacional, is structured to gauge the students' awareness and knowledge of what cyberbullying is. We submitted this updated survey to students of the Liceo Scientifico 'G. Keplero' in Rome, while also inviting them to provide their feedback on the study itself.

b) We reviewed and published the questionnaire as a preprint. The data we gathered strongly hinted at the need for surveys and informational material to use language and concepts adolescents could relate to.

c) In order to disseminate our results we will create programs focused on and carefully tuned to our reference population (teenagers) i.e. teacher workshops, informational posters, and extracurricular activities.
Results We were happy to find unexpected feedback; students were interested in the results of our survey. Both teachers $(60 \%)$ and students $(80 \%)$ showed a significant amount of interest in being advised on the outcome. Although the same sample population had been subject to several other questionnaires in the same timeframe, they only showed interest in following up on our results.

Conclusions Moving forward, we are now thinking of ways to share our results with the school community and start the third phase of the program. The next phase will focus on creating posters and informational materials on the most relevant points, as well as organizing teacher workshops in order to raise awareness on relevant topics.

\section{P308 AETIOLOGICAL ASSESSMENT OF PERMANENT CHILDHOOD HEARING IMPAIRMENT DIAGNOSED BY UNIVERSAL NEW-BORN HEARING SCREENING IN A PERIPHERAL IRISH HOSPITAL}

${ }^{1}$ Seamus Mac Farland*, ${ }^{2}$ Nick van der Spek. ${ }^{1}$ Paediatric Department, Cavan General Hospital, Cavan, Ireland; '2Paediatric Department, Cavan General Hospital , Cavan, Ireland

\subsection{6/archdischild-2019-epa.657}

Background and Aims The Universal New-Born Hearing Screening program (UNHS) was introduced in Cavan General Hospital in 2012. It aimed to identify newborns with Permanent Childhood Hearing Impairment (PCHI) and initiate early medical and educational interventions. In Ireland, PCHI affects 1-2 per 1,000 births and these children require a paediatric aetiological assessment. Our study aims to review the results of the aetiological assessments completed by paediatricians after identification by the UNHS of all infants with PCHI in our hospital. Currently,we use the British Association of Audiovestibular Physicians (BAAP) guidelines 2015 to aid our assessment.

Methods A retrospective chart review was carried out of patients with confirmed PCHI identified by the UNHS over a four-year period from October 2012 to November 2016. Data such as audiological diagnosis, investigations, and final aetiological diagnosis were recorded.

Results In total, 12 patients where identified (6 Male: 6 Female). On average, 3 cases per year of PCHI were diagnosed, which is in keeping with the birthrate in Cavan $(\sim 2,000$ per year).

\begin{tabular}{lllll} 
Abstract P308 Table 1 & \multicolumn{4}{l}{ Audiological Diagnosis } \\
\hline & $\begin{array}{llll}\text { Mild } \\
(20-40 \mathrm{~dB})\end{array}$ & $\begin{array}{l}\text { Moderate } \\
(40-70 \mathrm{~dB})\end{array}$ & $\begin{array}{l}\text { Severe/Profound } \\
(>70 \mathrm{~dB})\end{array}$ & Total \\
\hline Unilateral & 1 & 1 & 3 & 5 \\
Bilateral & 2 & 2 & 3 & 7 \\
Total & 3 & 3 & 6 & 12 \\
\hline
\end{tabular}

In our cohort, bilateral hearing loss $(n=7)$ was more common than unilateral $(n=5)$. In terms of severity, severe/profound hearing impairment $(n=6)$ was the commonest level of PCHI diagnosed.

Of the 12 patients identified, 3 were not assesed due to emigration and parental refussal. Of the 9 patients remaining, 7 were fully diagnosed (78\%) and 2 are still undergoing 
investigations. Of the 7 diagnosed: 3 had mutations in Connexin 26 gene (CNX), 2 had congenital cytomegalovirus (CMV) infections, and 2 had structural abnormalities of the innerear.

Conclusion In our small cohort, aetiological assessment was successful in establishing a diagnosis in $78 \%$ of cases of PCHI identified by the UNHS. The top 3 investigations to aid diagnosis include MRI Brain \& Internal Auditory Meati, Urinary CMV, andCNX 26 genetics.

\section{P309 THE CHALLENGE OF THE NOMADS}

Cillian Lineen*, Peter O'Reilly, Aisling O'Riordan, Katie Flinn, Alwyn Charles, AnneMarie Murphy. University Hospital Limerick, Limerick, Ireland

10.1136/archdischild-2019-epa.658

Background Irish Travellers are a Roman Catholic endogamous minority group with origins dating back to the twelfth century. Latest figures estimate their numbers at 40,129 on the Island of Ireland.

The Romani people colloquially known as the 'Roma Gypsies' are an indo-Aryan ethnic minority group originating from the northern Indian subcontinent, also traditionally itinerant and estimated to number 5000 in Ireland at any one time.

Often overlooked, nomadic races are unique minority groups with specific health needs

Aims Our objective was to investigate the knowledge and practices in relation to health and health intelligence of the modern nomadic races inhabiting our region with a particular focus on identifying key areas where intervention or education may reduce barriers and encourage access to healthcare, thus reducing health inequalities.

Methods A review of the current available literature and a database search were undertaken and relevant Clinicians and organisations contacted in an effort to compile data on these two nomadic groups to inform appropriate action in the area of Traveller and Roma Child Health

Results Nomadism is a core feature of their identity and involves more than just travel, the physical act of moving is just one aspect of a nomadic mindset that permeates every aspect of their lives. Nomadism entails a different way of looking at the world, a different way of perceiving things.

Conclusion Our study highlights the difficulties of healthcare provision for nomadic groups and provides useful epidemiological information with particular reference to the healthcare needs of 'invisible' minority groups marginalised in our modern Irish society.

\section{P310 HEALTH STATUS OF A GROUP OF CHILDREN IN A RESIDENTIAL CARE OF ROME}

${ }^{1}$ Pietro Ferrara*, ${ }^{2}$ Roberta Autuori, ${ }^{2}$ Alessandro Di Lucia, ${ }^{2}$ Flavia Dosa, ${ }^{2}$ Giulia Franceschini, ${ }^{2}$ Diletta Saitta, ${ }^{2}$ Michela Pulcino, ${ }^{2}$ Federica Di Ruscio, ${ }^{2}$ Maria Elisa Amodeo, ${ }^{3}$ Massimo Pettoello-Mantovani. 'Institute of Pediatrics, Catholic University Medical School, Rome, Italy; ${ }^{2}$ Campus Bio-Medico University, Rome, Italy; ${ }^{3}$ Department of Pediatrics, Scientific Institute Casa Sollievo della Sofferenza, University of Foggia, Foggia, Italy

\subsection{6/archdischild-2019-epa.659}

Objective To evaluate health status of a group of children placed in a residential care in the city of Rome.

Design It is a cross-sectional study.
Setting We drawn up a questionnaire, which was filled out anonymously by each educator with the data of the child of which each was responsible. Analyses were conducted using the Microsoft Excel 2016 program. We used descriptive statistics to describe patients' information.

Patients We selected the residential care Il Girotondo, located in Rome. In April 2018 it hosted 17 children, 8 boys (47\%) and 9 girls (52\%), with a mean age of $23.9 \pm 14.6$ months. $35 \%$ (6/17) of children was from Romania, 29\% (5/17) of children was from Italy, 18\% (3/17) was from Nigeria, 6\% (1/ 17) of children was of Rom ethnicity. One child had the father from Nigeria and the mother from Russia and one children had the father from Bulgaria and the mother from Algeria. The children were admitted to the residential care at a mean age of $15.7 \pm 15.1$ months. The mean length of stay was $8.4 \pm 5.2$ months.

Interventions Not applicable.

Main Outcome Measures Respiratory, urinary, intestinal, neurological, motor and psychological diseases, allergies to pollens or food, the state of vaccination, the re-lationship of the child with other children, the school attended and the academic achievement reported by their teachers.

Results Respiratory diseases were the most frequent (37.5\%), expecially in the fe-male group $(55.6 \%)$ than in the male group (14.3\%), followed by tendency to isola-tion (18.2\%), neurological diseases (17.8\%), not being at ease with other children (16.7\%), intestinal diseases (11.8\%), lack of acceptance by the others children $(8.3 \%)$, motor diseases $(6.3 \%)$ and psychological pathologies (5.9\%). About respira-tory diseases, asthmatic pathologies affected $12.5 \%$ of the sample and the $22.2 \%$ of the girls. No one had any urinary diseases or pollen and food allergies. All children were vaccinated. Lastly, $80 \%$ of the sample $(100 \%$ of males and $62.5 \%$ of females) did not attend any school. Two girls attended the day care and one girl attended the kindergarten.

Conclusions Beyond the psychological pathologies, the children hosted in the alternative care suffer from physical and development disease more than the general pediatric popu-lation. It is needful to perform a systematic analysis of the data to define the real needs for this special paediatric population in order to start them on an appropriate medical path.

\section{P311 RISK FACTORS OF RETARDATION THE FETAL DEVELOPMENT AND THEIR PREDICTORATIVE SIGNIFICANCE}

${ }^{1}$ Rusudan Karseladze*, ${ }^{1,2}$ Maka Chigladze, ${ }^{1}$ Liana Lorjoliani. ${ }^{1}$ Tbilisi State University, Fac. of Medicine, Tbilisi, Georgia; ${ }^{2}$ Clinic 'Pineo', Tbilisi, Georgia

\subsection{6/archdischild-2019-epa.660}

Search of the causes of formation of fetal development is a topical problem of perinatology. Distribution of fetal development is $10-44 \%$ on average. Delaying the development of the fetus by the American obstetrics in 2013 has been recognized as 'the most common and difficult problem of modern obstetrics' Nowadays there is enough information on the etiology and pathogenesis of impeding the development of the fetus. Here it should be noted that the risk factors are not completely systematized, the frequency and the share of each factor in the formation of syndrome.

The aim of the research was to study the mother's socialhygienic and medical-biological risk factors, determining their 